\title{
CLASH: Compliant Low cost Antagonistic Servo Hands
}

\author{
Werner Friedl, Hannes Höppner, Florian Schmidt, Máximo A. Roa, and Markus Grebenstein
}

\begin{abstract}
This paper presents the first two members of the new generation of CLASH hands, which exploit low cost actuation and rapid prototyping to create antagonistic modular and lightweight hands and grippers. The hands approach the robustness of the DLR Awiwi hand with a much lower complexity and cost. To reduce the number of required actuators, a differential coupling mechanism for underactuated fingers was developed, along with a new mechanism that uses variable stiffness actuation in order to increase the workspace of underactuated fingers. The hands provide a research platform for both hand-in-hand and robotic grasping. Design aspects are discussed, and an initial experimental validation verifies the hands' performance.
\end{abstract}

\section{INTRODUCTION}

Soft manipulation is a promising approach to attain robust grasping of objects while coping with uncertainties of real world scenarios. One of such scenarios is, for instance, the application of soft robotic hands and the exploitation of environmental constraints to automatize robotic grasping of groceries having different sizes, weights, shapes, and softness for online supermarkets. The combination of an inherent mechanical intelligence for adapting the shape without increasing the control complexity [1] and the smart usage of the stiff environment enables a safe and reliable grasp of objects while reducing the amount of necessary sensory information. Exploiting the possible interactions with the environment is an effective venue to cope with inherent uncertainty in real world scenarios, but this ability is limited by the hand morphology, control, and compliance [2].

Different soft robotic hand technologies with embodied compliance have emerged in recent years. While serial elastic hands [3] and hands with structural elasticity [2] have a rather fixed stiffness that changes with applied grip forces, hands such as the Awiwi hand [4] of the DLR Hand Arm System [5] have an additional motor for each degree of freedom (DoF) to further decouple stiffness from its increase with force. One advantage is that such hands can be stiff even when no grip forces are applied to an object. This can be especially helpful for lifting heavy but delicate objects such as fruits, where applied forces have to be limited in order to avoid damaging the object. However, hands such as the Awiwi hand (with 19-DoF, and driven by 38 motors in the forearm) which are built to answer a broad range of research questions, e.g., optimal hand kinematics as well as optimal kinematic and stiffness synergies, are far too complex and expensive to be used in a real industrial application.

This work has been funded by the European Commission's Eighth Framework Program, project SoMa (grant H2020-ICT-645599).

All authors are with the Institute of Robotics and Mechatronics, German Aerospace Center (DLR), Wessling, Germany. \{firstname.lastname\}edlr.de

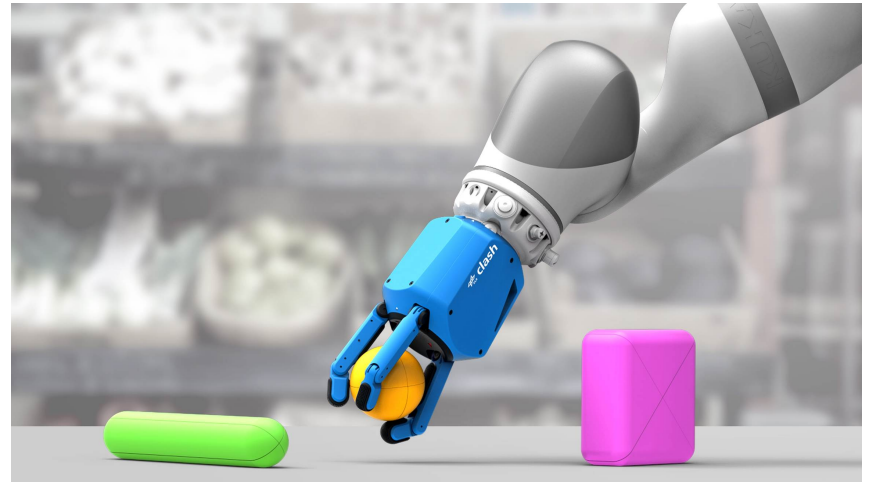

Fig. 1: The DLR CLASH 3F hand - A Compliant Low cost Antagonistic Servo Hand built for robotic grasping of delicate groceries.

This paper introduces and describes the evolutionary development of two soft robotic hands in a new family of DLR hands, generically called CLASH (Compliant Low cost Antagonistic Servo Hand), based on the technology used in the Awiwi hand [6]. The hands have three fingers -one versatile thumb and two opposing underactuated fingerscombined with low cost servo actuators and rapid prototyping parts, leading to a light, cheap, and robust platform for grasping experimentation. The DLR WHISG hand, described in Section II, is the first prototype of the CLASH family, and was mainly designed for investigating the variation of stiffness in humans during grasping. Section III presents grasping experiments with this hand. Section IV presents the DLR CLASH 3F, an enhanced version of the WHISG hand that includes essential improvements regarding hand aperture and force and stiffness behavior. Section V describes experiments with the CLASH 3F hand, and Section VI concludes the paper and discusses possible future work.

\section{The WHISG hand: A Wearable Hand to INVESTIGATE PASSIVE STIFFNESS IN GRASPING}

The WHISG hand is a wearable hand designed to investigate the role of passive stiffness in grasping. The hand consists of one main, versatile finger with 4-DoF, plus two underactuated, 3D-printed nylon-plastic fingers having 2DoF each (Fig. 2). The main finger is actuated by four servos and can be used as a thumb, with coupled distal (DIP) and proximal (PIP) interphalangeal joints. It has a $n-1+1$ tendon design, i.e., the finger is underactuated (-1) but has an additional motor to change its overall stiffness (+1). This means that the two DoF in the metacarpal joint (MCP) can be moved independently, while PIP and DIP are 


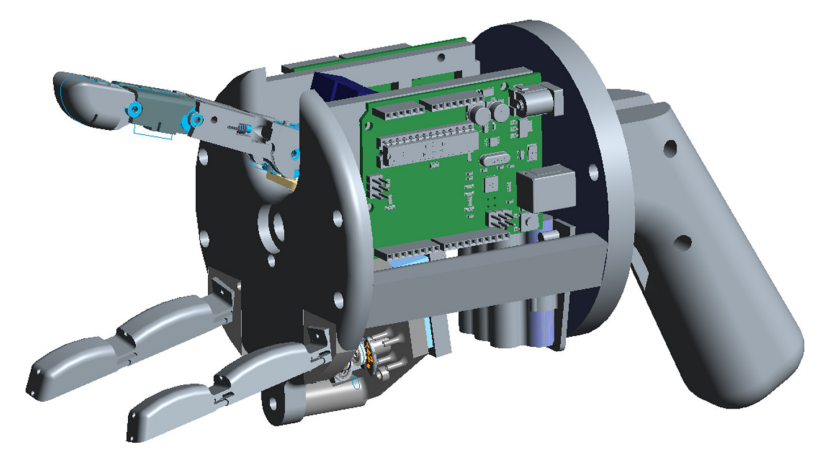

Fig. 2: The DLR WHISG hand: a Wearable Hand to Investigate passive Stiffness in Grasping.

differentially coupled. A return spring in the distal joint keeps the distal joint straight until the proximal joint gets in contact. The following matrix describes the chosen tendon coupling for the WHISG thumb, the radii in the first row control the abduction/adduction, in the second row the metacarpal and the third and fourth row the proximal and distal joint; columns correspond to tendons (for more details see [7]):

$$
R_{\text {WHISG thumb }}=\left(\begin{array}{cccc}
R_{4} & -R_{4} & 0 & 0 \\
R_{1} & R_{1} & -R_{6} & -R_{6} \\
0 & 0 & R_{2} & -R_{2} \\
0 & 0 & R_{3} & -R_{3}
\end{array}\right)
$$

All four tendons run over a so-called flexible antagonistic spring element (FAS) [6], which measures the tendon force and makes the fingers robust against external perturbations. A stiffness change always affects all four joints simultaneously. A rotatory spring is used (not a linear one), which provides a simpler form for the FAS used in the Awiwi hand. Four Bluebird BMS 385 servos actuate the main finger. With its maximum servo torque of $0.45 \mathrm{Nm}$, the finger can reach approximately $10 \mathrm{~N}$ fingertip force. The two other 2-DoF fingers have a $n / 2-1+1$ design, and they are driven by two antagonistic servos. Depending on the commanded direction, the servos change either position or stiffness of the two fingers (Fig. 3). Contrary to the concept of the Pisa/IIT softhand [8], the differential tendon mechanism is integrated in the servo winder. This means that each servo actuates one of two differential winders, such that it pretensions the tendon but is not rigidly fixed to it. Different to the thumb, in this case moving the fingers always requires to change the tendon force and not just the finger position, which causes the FAS springs to deflect. The maximum fingertip force of each finger is $2.5 \mathrm{~N}$. The benefit of this solution is that normal servos can be used, without changing its range of motion or the electronics.

The hand is additionally equipped with a handle that includes a trigger switch and an adjustment wheel, thus allowing a user to hand-held the device and grip objects. By turning the adjustment wheel, the stiffness can be set, while actuating the trigger closes the hand. This enables for example to easily investigate the role of stiffness in a user

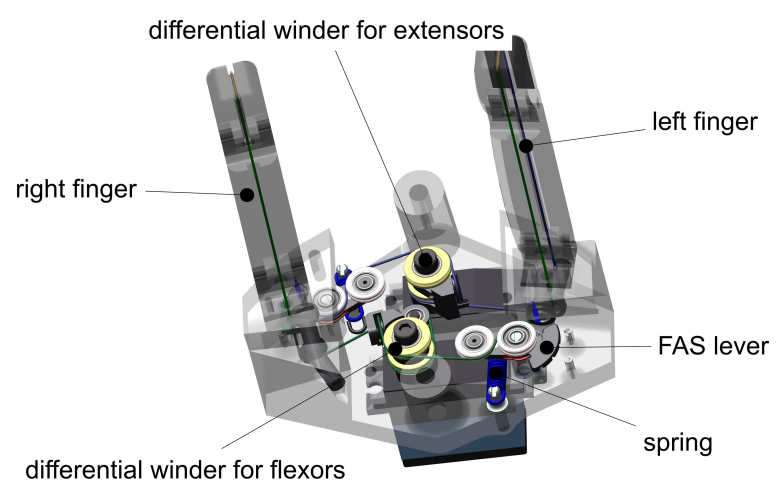

Fig. 3: Differential finger module of the WHISG hand. The flexor tendons are highlighted in green, and the extensor tendons in blue.

study with subjects grasping groceries, as described in the following section.

\section{EXPERIMENTS WITH THE WHISG HAND}

The use case selected for testing the CLASH hands is picking groceries out of a box, which is one of the use cases within the EU-project SoMa ${ }^{1}$ (Soft Manipulation). Fig. 4 presents some of the tests of the WHISG hand trying to pick different fruits and vegetables [9], [10], [11]. We found experimentally that the hand is able to grasp apples, bananas and cucumbers up to a weight of $500 \mathrm{~g}$, if the stiffness is set to its maximum using the adjustment wheel. When the stiffness is set to its minimum, which results in a pure power grasp, the hand is able to grasp bottles up to $1.1 \mathrm{~kg}$. This difference can be explained by the unsymmetrical stiffness range of the thumb flexor and extensor motors. Due to the $n+1$ design, the extensor reaches its force-limit at $20 \%$ of the force limit of the Metacarpal flexor.

This design provided sufficiently good hand performance for grasping fruits in the use case, while it was also possible to grasp smaller objects. However, a more detailed analysis of the two highly underactuated nylon fingers shows considerable limitations of the chosen design. Fig. 5 shows a force-stiffness diagram of one nylon-finger pushing on a force sensor, with the finger completely stretched, using stiffness pretensions between $10 \%$ and $50 \%$. As it can be seen, the measured resistance can be increased rather well at no load. However, as the applied force increases, the possible variation in stiffness is considerably reduced, since the measured resistance at no load is mainly provided by static friction. This limitation in stiffness-variation is caused by the underactuated design and differential joint coupling of the secondary fingers, in which the stiffness cannot be directly influenced as in the case of the thumb. Furthermore, the sensitivity of the force sensor is limited

\footnotetext{
${ }^{1}$ Project SoMa, Soft Manipulation, http://soma-project.eu/
} 

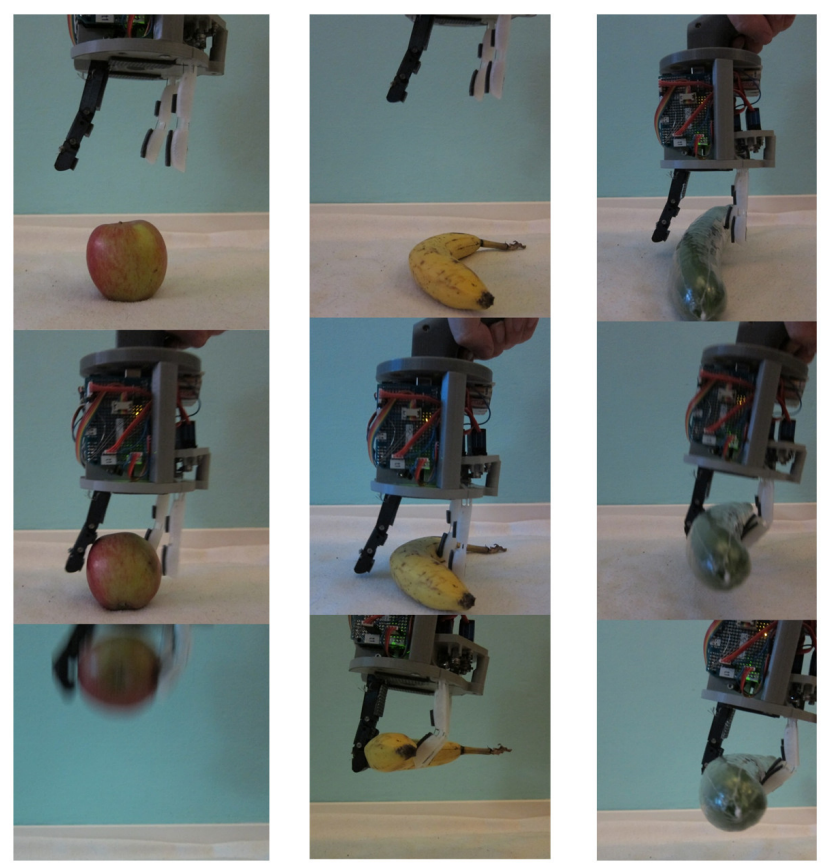

Fig. 4: Apples, bananas and cucumbers with a weight of up to $500 \mathrm{~g}$ grasped using the WHISG hand. The hand is held and operated by a human user.

in comparison to the thumb, because the FAS sensor has its highest force resolution for low forces, and gets always deflected when finger positions are changed. Due to these identified drawbacks of the highly underactuated fingers, we decided to improve their actuation mechanism and to add degrees of actuation and reduce underactuation in order to obtain better stiffness variation. Moreover, we found that the differential coupling between proximal and distal joint of the thumb also limits its variation in stiffness when pretensioning, as can be seen in Fig. 10. In consequence, we decided to make one more iteration to reduce the DoF of the thumb but still keeping the same number of motors.

\section{The CLASH 3F hand: A COMPliant Low COST ANTAGONISTIC SERVO HAND}

As a result of the knowledge gained with the WHISG hand, we defined the following requirements for a new design:

1) The hand kinematics must be optimized for the groceries of the use case.

2) Same actuator boxes have to be used for thumb and the two opposing fingers in order to increase system modularity.

3) Tendon coupling has to be optimized to get stronger fingers.

4) The design should enable an easy integration of different sensors for testing, e.g. tactile sensors.

5) The stiffness behavior of both thumb and fingers (Fig. 5) must be improved.

6) A force feedback handle rather than a pure trigger switch has to be integrated.

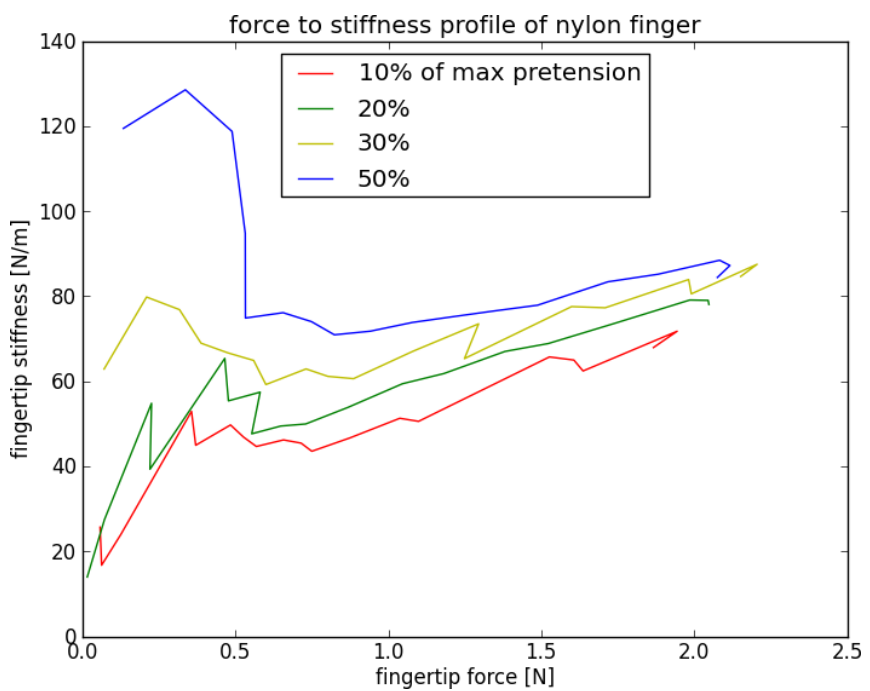

Fig. 5: Force-stiffness profile of the nylon fingers for different pre-tensions. While the change in measured resistance is quite promising for low forces, it becomes insufficient as force increases.
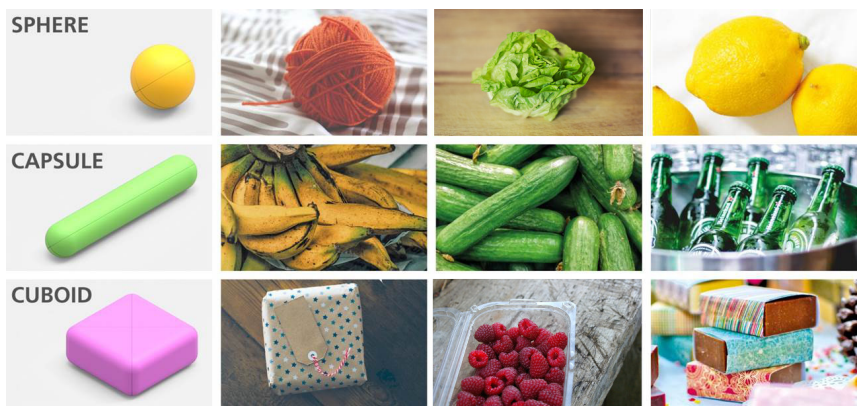

Fig. 6: Different groceries (iceberg lettuce, cucumber, blueberry boxes) and their simplification used for the optimization of finger segment lengths in the CLASH $3 \mathrm{~F}$ hand. The same primitive shapes can describe a large variety of objects.

7) The modular design should allow testing different finger configurations (e.g. 2- or 3-DoF, or lockmechanisms).

These requirements lead to the design of a new hand prototype with three fingers, hereafter called CLASH $3 \mathrm{~F}$ gripper. It provides a research platform for both hand-inhand grasping and for doing grasp experiments with robots.

\section{A. Kinematic optimization}

The CLASH hand has been designed considering a geometrical optimization, including requirements on force closure for grasping selected objects from the use case. These objects are an iceberg lettuce (approximated by a sphere), a box of blueberries (approximated by a cuboid) and a cucumber (approximated by a capsule), as illustrated in Fig. 6. Due to the problems already described with the stiffness variation for the WHISG hand fingers, we decided to first investigate a kinematics with no proximal joints, hence the new version of the fingers has two DoF for the 


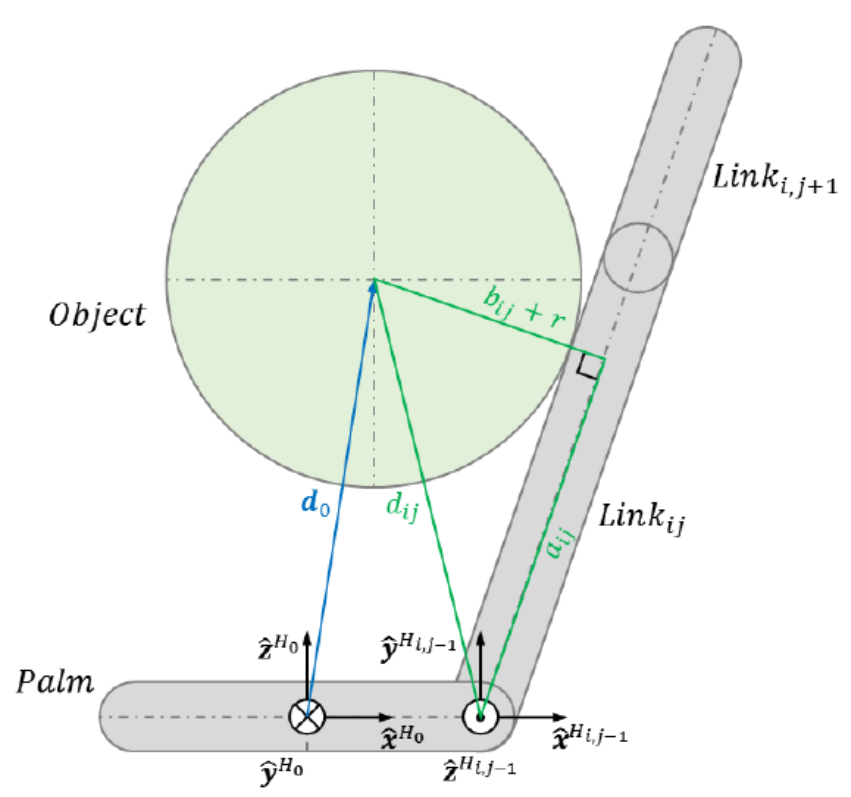

Fig. 7: Kinematic optimization of the fingers (segment lengths and length of the palm).

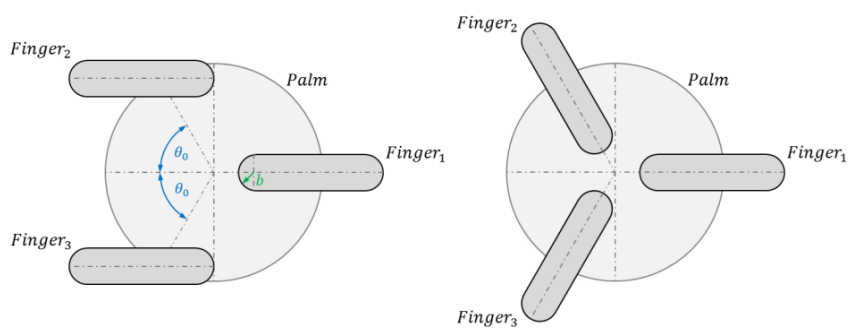

Fig. 8: Kinematic optimization of the hand (finger placement and orientation).

fingers and 3 DoF for the thumb. The geometrical design was performed in a two-stage process. In the first stage, the segment lengths and the length of the palm for a planar hand (Fig. 7) were selected by optimizing for maximization of the reachable and force closure space, i.e. trying to have the largest possible workspace that guarantees force closure grasps on the selected objects. In the second stage, the placement and orientation of the fingers were decided using the same optimization process (Fig. 8). The final design resulted in having fingers pointing toward the center of the palm.

\section{B. Design of the differential fingers}

To improve the modularity and capabilities of the hand, CLASH 3F uses the same servo module with four motors for the thumb and for the differential fingers. The two extra servos of the module are used to increase the fingertip force, the stiffness-variation capabilities, and the reachability. The MCP joints of the secondary (differential) fingers can be actuated independently, but the proximal joints of the two fingers are coupled, similar to the coupling that happens between human pinky and ring fingers. In this way, the

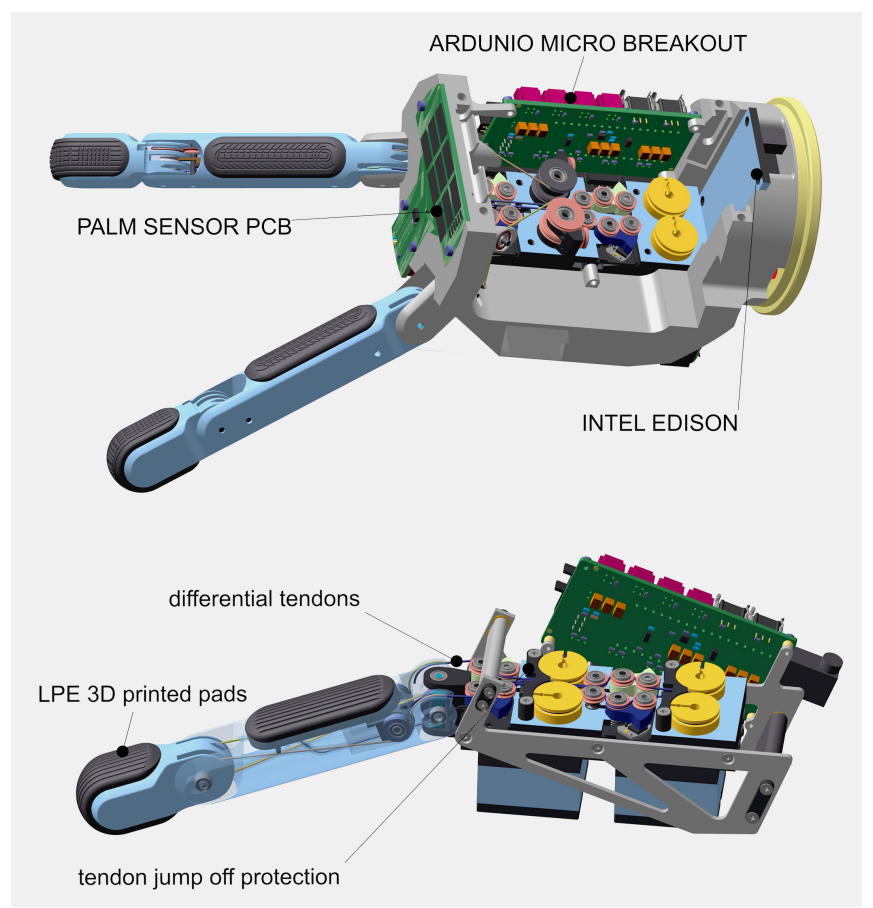

Fig. 9: Thumb and differential finger module of CLASH 3F.

fingers have 3 active DoF due to the reuse of the differential modules of the WHISG hand. The chosen tendon coupling for the 2 DoF version of the secondary fingers is:

$$
R_{\text {differential fingers }}=\left(\begin{array}{cccc}
-R_{1} / 2 & R_{3} / 2 & 0 & R_{2} \\
-R_{1} / 2 & R_{3} / 2 & R_{2} & 0 \\
-R_{4} & R_{5} & 0 & 0
\end{array}\right)
$$

With the new design, the maximum fingertip force is now $10 \mathrm{~N}$ for these fingers.

\section{Thumb design}

Due to the increased fingertip force of the differential fingers, the thumb was redesigned to increase its force capabilities by a factor of two. Inspired by human tendon routing, the two flexor tendons for the MCP joint end now at the proximal joint. In the human, the strongest finger tendon is the profundus tendon, which ends at the distal bone and can generate torque on all prior joints. This approach is also used by all tendon-driven underactuated hands, but only with one tendon. The differential coupling between the joints improves the capabilities to deal with environmental constraints, in particular for sliding the fingertip over a surface to reach flat objects, as it reduces the control requirements due to the finger self-adaptation. In other words, this allows an open-loop control of the sliding motion if the extensors are controlled by a soft admittance control. On the other side, this routing leads to a very strong proximal joint (PIP) and a weak MCP joint. To solve this situation, the proximal extensor tendon is used as flexor in the MCP joint, as 
described by the tendon coupling matrix:

$$
R_{\text {CLASH thumb }}=\left(\begin{array}{cccc}
R_{4} & -R_{4} & 0 & 0 \\
R_{1} & R_{1} & -R_{5} & R_{3} \\
R_{2} & R_{2} & 0 & -R_{2}
\end{array}\right)
$$

If we compare the tendon coupling with the WHISG hand (see Eq. 1), we see now two tendons that work in the base and the distal joint as flexors, and the extensor of the distal joint acts also as flexor in the base. This design doubles the maximum fingertip force up to $20 \mathrm{~N}$, and considerably increases the range of stiffness variation under load in comparison to the WHISG hand, as shown in Fig. 10. The result looks qualitatively similar to the force-stiffness diagram measured at the human pinch grip (see Fig. 11).
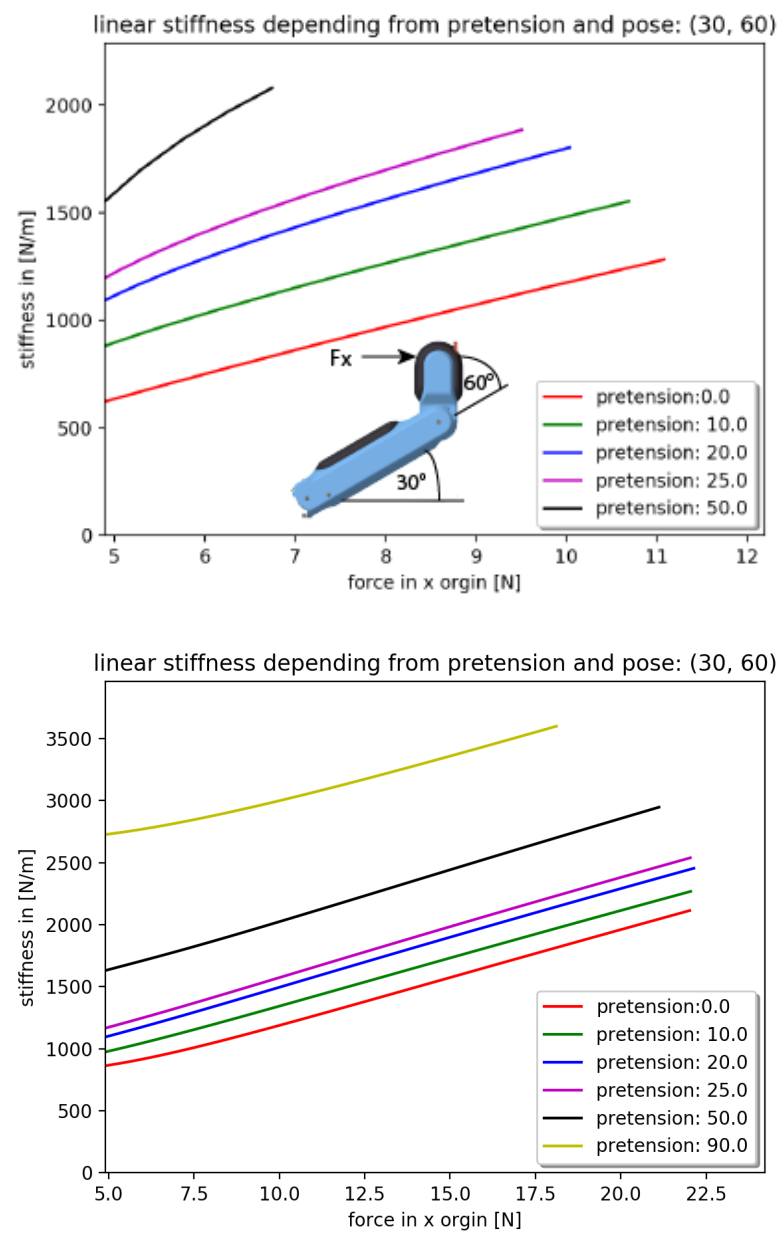

Fig. 10: Comparison of force-stiffness profiles of WHISG and CLASH thumb. Start position: base $30^{\circ}$, distal $60^{\circ} ; 5 \mathrm{~N}$ in $\mathrm{x}$ direction are generated by the springs, then the finger is deflected along the $\mathrm{x}$ direction until the first tendon reaches the maximum force of $65 \mathrm{~N}$, force in y direction is zero. Repeated with increasing pretension in $10 \%$ increments.

To analyze the hand behavior when grasping an object, a simple planner was used to compute grasp forces required to grab a sphere from the top, with density equal to water (because most fruits and vegetables have a density similar

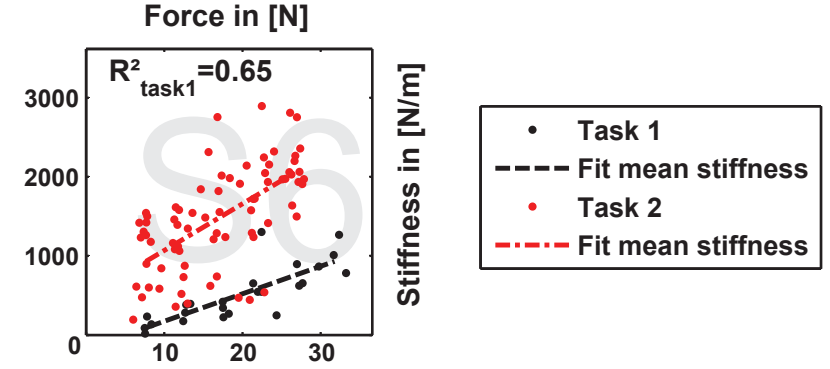

Fig. 11: Exemplary diagram of human grip stiffness, adapted from [12].

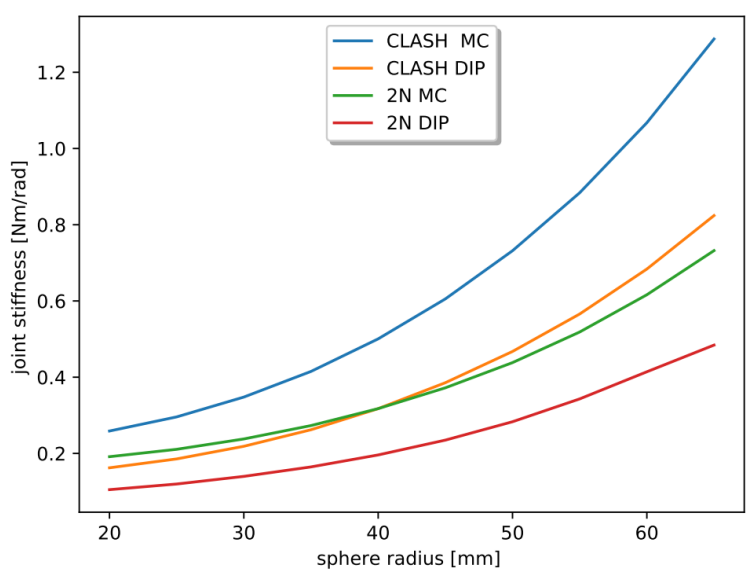

Fig. 12: Comparison of object-dependent stiffness between CLASH and a $2 n$ design.

water, e.g. peaches, apples, cucumbers and mangoes can swim - potatoes and tomatoes sink). This leads to the computation of an object-dependent stiffness, as shown in Fig. 12. The figure shows that the stiffness for the CLASH hand increases faster than for example the $2 n$ coupling of the 3-DoF fingers, which are used in the two-finger hand of the DLR Hand Arm System. The result for the WHISG hand is not presented, but it is quite similar to a $2 n$ design. The faster increase of stiffness results in a more stable grasp against weight variations of the gripped object.

\section{Finger with locking mechanism}

The identified drawbacks of the highly underactuated nylon-fingers of the WHISG hand gave us the idea of locking single joints for such fingers, which can also easily be tested with the new design. The idea of locking one or more joints of an underactuated finger has already been used in different designs. For instance, Aukes et al. [13] presented a design that can selectively lock single DoFs of a serial-elastic hand using electrostatic braking at the joints. A different solution is to change the stiffness of a single joint by heating up a shape memory polymer (SMP) [14]. Both solutions need extra actuators to lock or change the stiffness of the joints, which leads to an increase of system complexity. Additionally, 


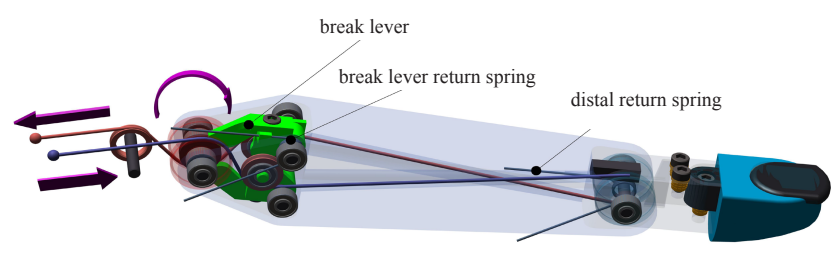

a) normal finger motion, by pulling on flexor and releasing extensor - moves base joint. Distal return spring keeps distal joint in position. The return springs of break levers keep them fixed. break lever press on pin and block base

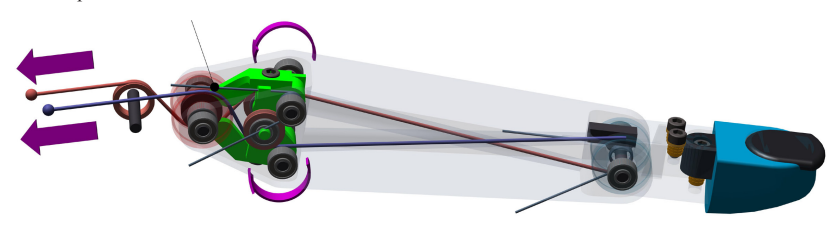

b) locking base joint by pulling on both tendons, result in rotation of break levers and pushing them against base pin, which is fixed to the base - locking base joint.

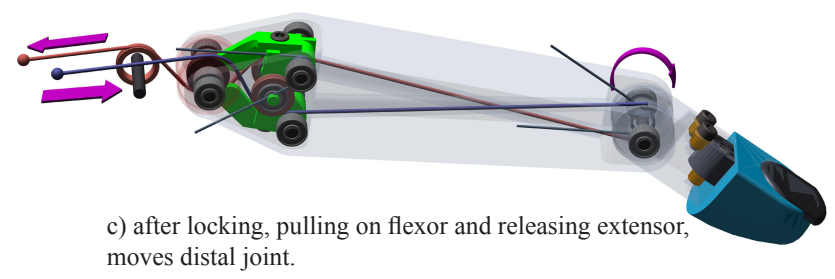

Fig. 13: Prototype of finger with stiffness joint break. The finger has two DoF and is driven by two motors. When pretensioning is applied to the elastic elements in the base of the hand, the lever arms are deflected and hinder the base joint in its movement, thus allowing the motion of the distal link.

varying the stiffness by SMP is very slow, and modifies the natural motion of the finger. The idea of the finger presented here is to use the variable stiffness actuation to lock the joints (Fig. 13). The finger has two DoF and is driven by two motors coupled with FAS elements. Attached to the finger base there are two lever arms, which decouple the base joint when deflected. A spring between these beams pushes them into the position where no breaking force is applied to the base joint. As the tendon force and stiffness increase, the distance between the lever pulleys is reduced, hence the friction increases such that the base joint is hindered from moving. As a result, the finger movement, e.g. the ratio in movement between both joints at no load, can be varied by changing the pretension.

In normal operation the finger behaves like an underactuated finger: if both motors move the finger, the base joint flexes until the base joint gets in contact with an object, or it reaches its end-stop, and then the distal joint moves. Contrary to the DIP joint, the base joint needs no return spring because the second motor is the extensor. This finger concept is going to be used in the next evolution of the CLASH 3F hand.

\section{E. Electronics, sensor and software concept}

To get a simple and low cost system, both hands use Arduinos to collect sensor data and control the servos (see Fig. 14). The two Arduino Micros (Atmel Mega32U4) can control up to five servos with their timers. The potentiometer values of the servos are fed back to the Arduinos to calculate all finger positions. The angles of the variable stiffness levers are measured by the ICHaus MP, an analog Hall sensor, which works well with the magnet from the Awiwi FAS. Both Arduinos communicate with each other via their SPI interface. The SPI master has a USB connection to a Linux computer, which in case of the portable version of the CLASH is an Intel Edison board. This board runs the executable code generated from the Simulink model, a driver process handling the USB communication, and a web server providing parameter access. Inter-process communication is provided by the DLR Links_and_Nodes communication middleware. However, in the portable version of the WHISG hand all software is implemented on a microcontroller (see Videos in [9] and [11]), and the hand is controlled via the pistol knob. The USB communication uses synchronous transfers with a payload length of 32 bytes in both directions, at a rate of $1 \mathrm{kHz}$.

The palm is equipped with a sensor board that has an IMU to get the orientation of the hand, a proximity sensor to detect objects before contact, and a small microcontroller, Atmel Tiny1634, to collect data from palm and fingertip touch sensors. The touch sensor is based on the piezoresistive effect of the 3M Velostat foil or ESD foam [15]. The palm has a $3 \times 3$ sensor area, and the fingers can be equipped with $3 \times 3$ fingertip sensors. The sensor board is connected via $\mathrm{I} 2 \mathrm{C}$ to the thumb Arduino board. In normal operation, the three $\mathrm{I} 2 \mathrm{C}$ sensors are read at a rate of $166 \mathrm{~Hz}$. In case fast tactile information is necessary, the point of interest can be switched so that touch information can poteentially be updated at $500 \mathrm{~Hz}$. Additional sensors can still be connected: the SPI master has a free I2C and UART port which could for example be used to integrate the SPAKFUN Robotic Finger Sensor [16]. A big advantage of using Arduino boards is that software for such sensors is freely available and can easily be integrated.

\section{F. Comparison of CLASH $3 F$ with respect to other DLR hands}

Table I presents a comparison of different finger features among a number of DLR hands. The comparison is performed at finger level, because from the modular construction principle it is possible to build a hand with a similar kinematic structure to the DLR Hand II or the DLR/HIT Hand using the thumb module of the CLASH 3F. It would also be possible to build a Awiwi-inspired hand using the new fingers plus a forearm. 


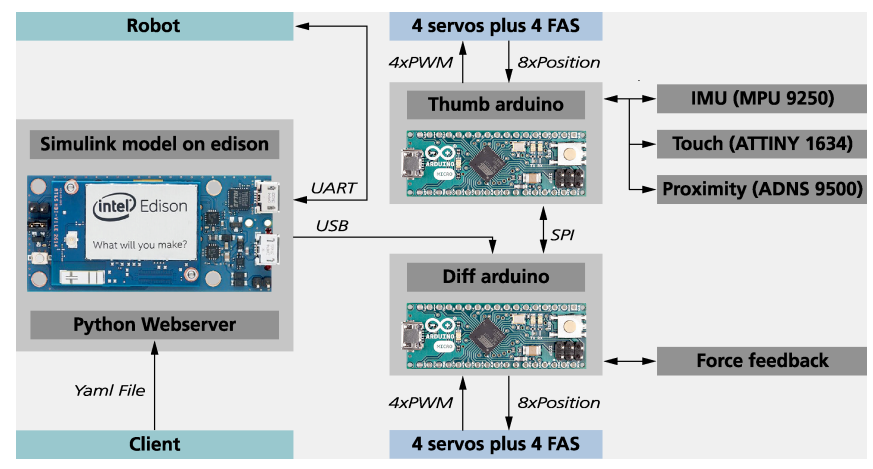

Fig. 14: Electronic design of CLASH 3F.

TABLE I: Finger properties for different DLR hands

\begin{tabular}{|l|l|l|l|l|} 
Hand & $\begin{array}{l}\text { Fingertip } \\
\text { force [N] }\end{array}$ & $\begin{array}{l}\text { Module } \\
\text { weight [g] }\end{array}$ & Robustness & $\begin{array}{l}\text { Approx. } \\
\text { Cost [€] }\end{array}$ \\
\hline $\begin{array}{l}\text { Hand II [17] } \\
\text { Hit Hand II }\end{array}$ & 25 & 408 & 0 & 2000 \\
{$[18]$} & 250 & 0 & 2000 \\
DEXHAND & 25 & 440 & 0 & 3000 \\
{$[19]$} & & & & \\
Awiwi II & 20 & 700 & + & 16000 \\
WHISG & 10 & 200 & + & 300 \\
CLASH 3F & 20 & 200 & + & 300
\end{tabular}

1 AWIWI hand with steel tendons [7]

\section{EXPERIMENTS WITH THE CLASH 3F}

The experiments with the CLASH hand were performed with the hand mounted on a robot, to grasp fruits and vegetables. The experiments are shown in the video that accompanies this paper, and several snapshots are shown in Fig. 15. The fruits used in the experiment are printed plastic fruits and wood dummies. The plastic fruits were wrapped in cling film to get a more realistic friction behavior. The hand was also tested for grasping several objects of the YCB dataset [20], as shown in Fig. 16.

For the tested objects we adapted only the pre-grasp pose of the fingers, the force threshold and grasp stiffness were similar. These parameters should be decided online by a suitable grasp planner, based on the object weight and pose.

The video also shows preliminary results of the in-hand manipulation capabilities of the hand while grasping a plastic box with blueberries. This test shows that friction differences in the differential finger are not beneficial for a stable movement. The plastic box is hard to detect by standard vision procedures due to the reflectivity of the package, so the proximity sensor in the hand was used to detect the edges of the box. Fig. 17 shows the result for a linear motion over the box, performed to detect the edge. Tactile information was also used to detect the edge by following the torque profile of the finger, in this case, the thumb sliding over the object. A more extensive analysis of the in-hand manipulation capabilities of the hand is left a future work.

\section{CONCLUSION}

This paper introduced a new family of low cost soft robotic DLR hands, generically called CLASH (Compliant Low cost
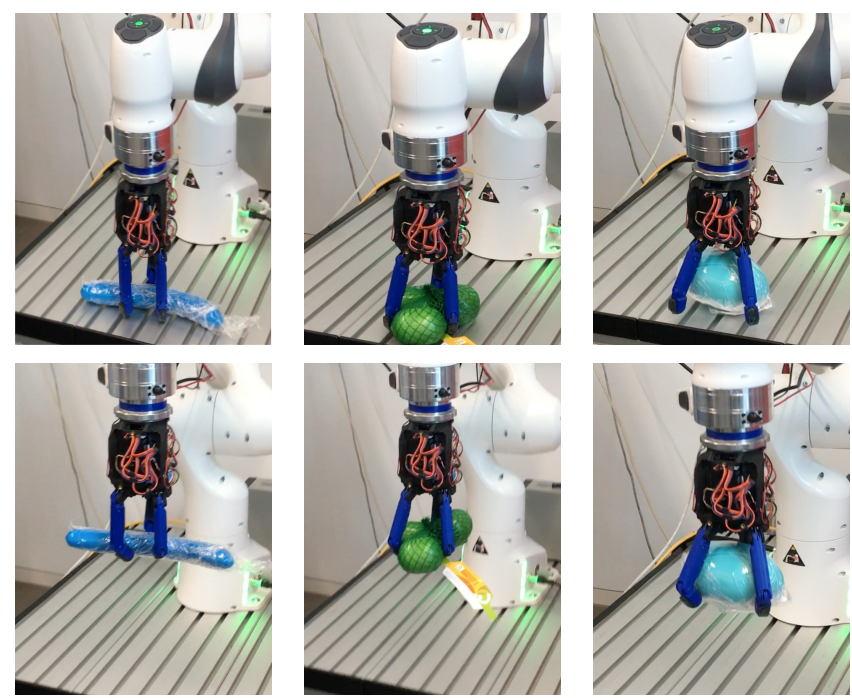

Fig. 15: Exemplary cucumber, kiwis, and mango with a weight of up to $500 \mathrm{~g}$ grasped with the CLASH $3 \mathrm{~F}$ hand.
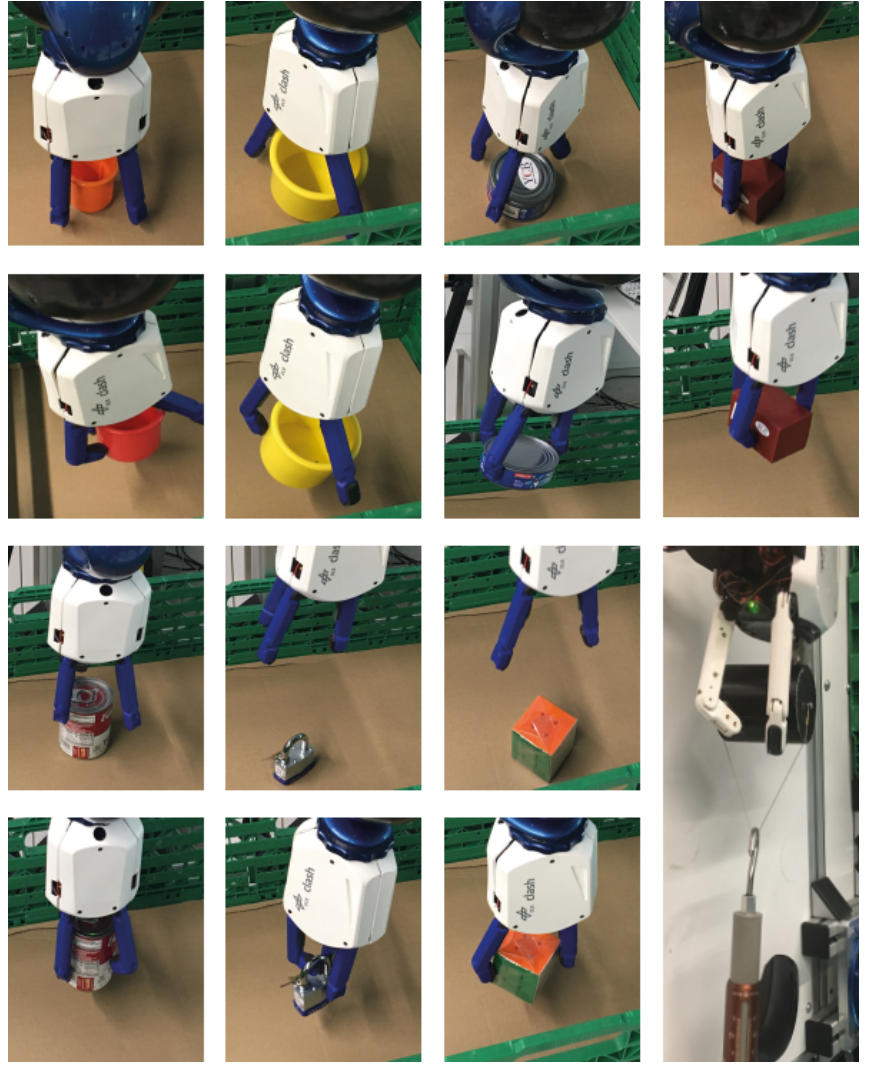

Fig. 16: Grasping household objects from the YCB object dataset. The lower-right picture shows a pull out test for a cylinder with diameter $60 \mathrm{~mm}$, resulting in a pullout force of $28 N$. 


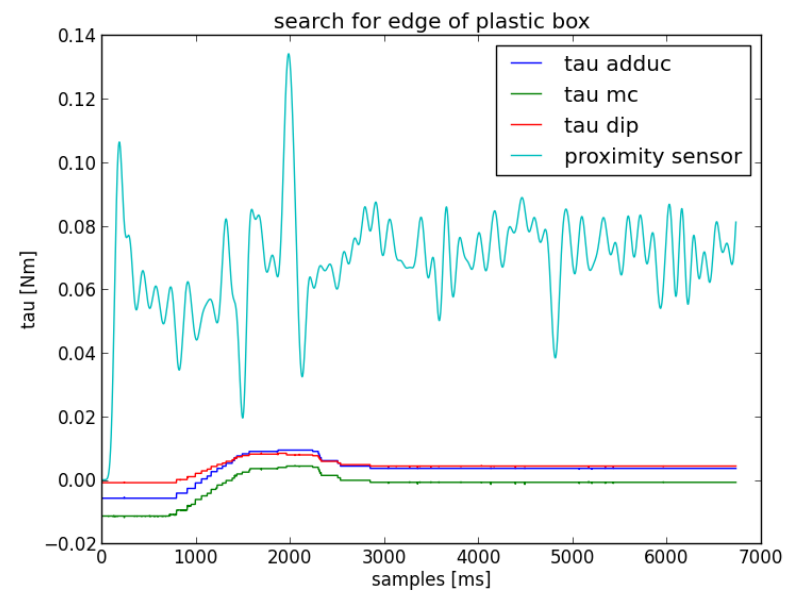

Fig. 17: Tactile and optical edge detection of a plastic box.

Antagonistic Servo Hand), based on the soft technology used in the Awiwi hand. They allow grasping a variety of fruits and vegetables defined for the use case of a logistic center that handles groceries, by providing variable stiffness behavior that can be tuned according to the characteristics of the grasped object. The hands can also be used to implement grasping strategies where the hand exploits contact with the environment, as demonstrated in the attached video. The hand is highly performant at a very low cost: the total material cost is less than $300 €$ due to its low cost actuators, electronics and 3D-printed fingers and structure. Our initial tests show a good hand performance. Extensive testing to verify the advantages of low-cost variable stiffness actuation for robot hands are an interesting line of research, currently ongoing at DLR.

\section{ACKNOWLEDGMENT}

The authors would like to thank Tilo Wüsthof for his assistance in designing the housings, Nikolaus Seitz for the layout of PCB electronics, Igor Merkowski for his contribution to the hand design, and people working at the DLR workshop for their great support during the construction process.

\section{REFERENCES}

[1] C. Eppner, R. Deimel, J. Alvarez-Ruiz, M. Maertens, and O. Brock, "Exploitation of Environmental Constraints in Human and Robotic Grasping," Int. J. Robotics Research, vol. 34, no. 7, pp. 1021-1038, 2015.

[2] R. Deimel and O. Brock, "A novel type of compliant and underactuated robotic hand for dexterous grasping," Int. J. Robotics Research, pp. 1$25,2015$.
[3] M. Tavakoli and A. de Almeida, "Adaptive under-actuated anthropomorphic hand: ISR-SoftHand," in IEEE/RSJ Int. Conf. Intelligent Robots and Systems, 2014, pp. 1629-1634.

[4] M. Grebenstein, Approaching Human Performance: The FunctionalityDriven Awiwi Robot Hand. Springer, 2014.

[5] M. Grebenstein, A. Albu-Schäffer, T. Bahls, M. Chalon, O. Eiberger, W. Friedl, R. Gruber, S. Haddadin, U. Hagn, R. Haslinger, H. Höppner, S. Jörg, M. Nickl, A. Nothhelfer, F. Petit, J. Reill, N. Seitz, T. Wimböck, S. Wolf, T. Wüsthoff, and G. Hirzinger, "The DLR hand arm system," in IEEE Int. Conf. Robotics and Automation, 2011, pp. 3175-3182.

[6] W. Friedl, M. Chalon, J. Reinecke, and M. Grebenstein, "FAS-A flexible antagonistic spring element for a high performance overactuated hand," in IEEE/RSJ Int. Conf. Intelligent Robots and Systems, 2011, pp. $1366-1372$.

[7] — "Frcef: The new friction reduced and coupling enhanced finger for the awiwi hand," in 2015 IEEE-RAS 15th International Conference on Humanoid Robots (Humanoids), 2015, pp. 140-147.

[8] M. Bonilla, E. Farnioli, C. Piazza, M. Catalano, G. Grioli, M. Garabini, M. Gabiccini, and A. Bicchi, "Grasping with soft hands," in IEEE-RAS Int. Conf. Humanoid Robots, 2014, pp. 581-587.

[9] W. Friedl. (2017) WHISG - a wearable hand to investigate passive stiffness while grasping. [Online]. Available: https://youtu.be/ IpJ0K9IVCDs

[10] F. Herold and W. Friedl. (2017) DLR low cost hand WHISG mounted on LBR robot. [Online]. Available: https://youtu.be/dmwzx-gsq0A

[11] W. Friedl. (2015) Test of different control modes with the thumb module of the DLR WHISG hand. [Online]. Available: https://youtu.be/IpJ0K9IVCDs

[12] H. Höppner, G. Stillfried, M. Große-Dunker, J. Bayer, and P. van der Smagt, "Key insights into hand biomechanics: Human Grip Stiffness Can Be Decoupled from Force by Cocontraction and Predicted from Electromyography," Frontiers in Neurorobotics, vol. 11, no. 17, pp. 1 $-20,2017$.

[13] D. Aukes, S. Kim, P. Garcia, A. Edsinger, and M. Cutkosky, "Selectively compliant underactuated hand for mobile manipulation," in IEEE Int. Conf. Robotics and Automation, 2012, pp. 2824-2829.

[14] Y. Yang and Y. Chen, "3D printing of smart materials for robotics with variable stiffness and position feedback," in IEEE Int. Conf. Advanced Intelligent Mechatronics, 2017, pp. 418-423.

[15] R. Koiva, M. Zenker, C. Schürmann, R. Haschke, and H. J. Ritter, "A highly sensitive 3D-shaped tactile sensor," in IEEE/ASME Int. Conf. Advanced Intelligent Mechatronics, 2013, pp. 1084-1089.

[16] R. Patel and N. Correll, "Integrated force and distance sensing for robotic manipulation using elastomer-embedded commodity proximity sensors," in Robotics: Science and Systems, 2016.

[17] J. Butterfass, M. Grebenstein, H. Liu, and G. Hirzinger, "DLR-hand II: next generation of a dextrous robot hand," in IEEE Int. Conf. Robotics and Automation, vol. 1, 2001, pp. 109-114.

[18] H. Liu, K. Wu, P. Meusel, N. Seitz, G. Hirzinger, M. H. Jin, Y. W. Liu, S. W. Fan, T. Lan, and Z. P. Chen, "Multisensory five-finger dexterous hand: The DLR/HIT hand II," in IEEE/RSJ Int. Conf. Intelligent Robots and Systems, 2008, pp. 3692-3697.

[19] M. Chalon, A. Wedler, A. Baumann, W. Bertleff, A. Beyer, J. Butterfaß, M. Grebenstein, R. Gruber, F. Hacker, E. Krämer, K. Landzettel, M. Maier, H. J. Sedlmayr, N. Seitz, F. Wappler, B. Willberg, T. Wimböck, G. Hirzinger, and F. Didot, "Dexhand: A space qualified multi-fingered robotic hand," in IEEE Int. Conf. Robotics and Automation, 2011, pp. 2204-2210.

[20] B. Calli, A. Walsman, A. Singh, S. Srinivasa, P. Abbeel, and A. M. Dollar, "Benchmarking in manipulation research: Using the YaleCMU-Berkeley object and model set," Robotics and Automation Magazine, vol. 22, no. 3, pp. 36-52, 2015. 